\title{
On the Analysis of Certain Flotation Processes with Velocities Depending on Time and Height of the Column
}

\author{
Daniela D. Parashkevova
}

\begin{abstract}
The present paper is an extension of the previous paper of the author where the flotation column dynamics has been investigated. Here we consider the case when particle sedimentation rate and bubble lifting speed depend on time and position in the column. We use the methods for examining the transmission lines set out in the papers mentioned in the References. We formulate a mixed problem for the system describing the processes in the column and present it in a suitable operator form. Then we prove an existence - uniqueness of generalized solution by the fixed point method. We show an explicit approximated solution as a step in the sequence of successive approximations.
\end{abstract}

Index Terms - Camera flotation, Fixed point method, Hyperbolic system, Successive approximations.

\section{INTRODUCTION}

The present paper continues investigations of the previous paper [1]. In [1] we have studied a system describing camera flotation process considered in [2]. Here we extend the results from [1], considering more complicated case when the velocities $V_{B}$ and $V_{P}$ depend on the one space variable and time, that is, $V_{B}=V_{B}(x, t)$ and $V_{P}=V_{P}(x, t)$. From mathematical point of view, the system describing flotation process remains of hyperbolic type. But this leads to more complicated type of the characteristics of the hyperbolic system. We study the following system:

$$
\begin{gathered}
\frac{\partial C_{B}(x, t)}{\partial t}=k_{1} C_{P}(x, t)-k_{2} C_{B}(x, t)-V_{B}(x, t) \frac{\partial C_{B}(x, t)}{\partial x}- \\
-C_{B} \frac{\partial V_{B}(x, t)}{\partial x} \\
\frac{\partial C_{P}(x, t)}{\partial t}=-k_{1} C_{P}(x, t)+k_{2} C_{B}(x, t)+ \\
+V_{P}(x, t) \frac{\partial C_{P}(x, t)}{\partial x}+C_{P} \frac{\partial V_{P}(x, t)}{\partial x}
\end{gathered}
$$

in the domain

$(x, t) \in \Pi=\left\{(x, t) \in R^{2}:(x, t) \in[0, H] \times[0, T]\right\}$. Here $C_{P}(x, t)$ is the mineral concentration in the liquid, $C_{B}(x, t)$ is the mineral concentration on the bubbles, $k_{1}$ and $k_{2}$ are

Submitted on August 17, 2021.

Published on September 07, 2021

Daniela D. Parashkevova, University of Mining and GeologyS t. I. Rilski”, Faculty of Mining Electromechanics, Department of Automation of Mining Production, 1700 Sofia, Bulgaria.

(e-mail: dani.parashkevova@gmail.com) prescribed kinetic constants describing particle transitions from one phase to another, $H>0$ is the height of the camera (column) and $[0, T]$ is prescribed time interval; $V_{P}(x, t)>0$ is a particle sedimentation rate, $V_{B}(x, t)>0$ - the bubble lifting speed. We note that $V_{B} \gg V_{P}$ (cf. [2]). The process in the camera are such that $V_{P}(x, t)$ is directed from the top to the bottom, while $V_{B}(x, t)$ the speed of the bubbles is directed upwards. That is why, the signs of $V_{B}=V_{B}(x, t)$ and $V_{P}=V_{P}(x, t)$ are opposite ones in (1).

For the system (1) can be formulated the following mixed (initial-boundary value) problem: to find the unknown concentration functions $C_{P}(x, t)$ and $C_{B}(x, t)$ in $\Pi$ satisfying initial conditions

$C_{B}(x, 0)=0, C_{P}(x, 0)=C_{P 0}$,

where $C_{P 0}=$ const. $>0$ is a prescribed initial concentration and boundary conditions

$C_{B}(0, t)=0, C_{P}(0, t)=C_{P 0}=$ const. $>0 \quad t \in[0, T]$.

In [3] the mathematical methods for investigation of transmission lines and some applications (cf. also [4] - [6]) are developed. We have applied these methods to our flotation problem [1]. Here we continue our investigations to more general case of flotation processes. We present the mixed problem for the above hyperbolic system in an operator form. Choosing a suitable function space, we prove existence theorems for (1) - (3) by the method from [3] - [6]. Finally, we demonstrate a simple way to obtain successive approximations tending to the solution of our problem.

Introduce denotations

$U=\left[\begin{array}{l}C_{B}(x, t) \\ C_{P}(x, t)\end{array}\right], \quad \frac{\partial U}{\partial t}=\left[\begin{array}{l}\frac{\partial C_{B}(x, t)}{\partial t} \\ \frac{\partial C_{P}(x, t)}{\partial t}\end{array}\right], \quad \frac{\partial U}{\partial x}=\left[\begin{array}{l}\frac{\partial C_{B}(x, t)}{\partial x} \\ \frac{\partial C_{P}(x, t)}{\partial x}\end{array}\right]$, 
$A=\left[\begin{array}{cc}V_{B}(x, t) & 0 \\ 0 & -V_{P}(x, t)\end{array}\right]$

Then (1) can be rewrite as

$$
\begin{array}{r}
\frac{\partial C_{B}(x, t)}{\partial t}+V_{B}(x, t) \frac{\partial C_{B}(x, t)}{\partial x}=k_{1} C_{P}(x, t)-k_{2} C_{B}(x, t)- \\
-C_{B}(x, t) \frac{\partial V_{B}(x, t)}{\partial x} \\
\frac{\partial C_{P}(x, t)}{\partial t}-V_{P}(x, t) \frac{\partial C_{P}(x, t)}{\partial x}=-k_{1} C_{P}(x, t)+k_{2} C_{B}(x, t)+ \\
+C_{P}(x, t) \frac{\partial V_{P}(x, t)}{\partial x}
\end{array}
$$

or in a matrix form

$$
\begin{aligned}
& {\left[\begin{array}{c}
\frac{\partial C_{B}(x, t)}{\partial t} \\
\left.\frac{\partial C_{P}(x, t)}{\partial t}\right]
\end{array}\right]+\left[\begin{array}{cc}
V_{B} & 0 \\
0 & -V_{P}
\end{array}\right]\left[\begin{array}{l}
\frac{\partial C_{B}(x, t)}{\partial x} \\
\frac{\partial C_{P}(x, t)}{\partial x}
\end{array}\right]=} \\
& =\left[\begin{array}{cc}
-k_{2}-\frac{\partial V_{B}(x, t)}{\partial x} & k_{1} \\
k_{2} & -k_{1}+\frac{\partial V_{P}(x, t)}{\partial x}
\end{array}\right]\left[\begin{array}{l}
C_{B}(x, t) \\
C_{P}(x, t)
\end{array}\right]
\end{aligned}
$$

or

$$
\frac{\partial U}{\partial t}+A \frac{\partial U}{\partial x}=\Gamma U
$$

$$
\text { Here the matrix } A=\left[\begin{array}{cc}
V_{B}(x, t) & 0 \\
0 & -V_{P}(x, t)
\end{array}\right] \text { is in a diagonal }
$$
form. Therefore the characteristic roots are $\lambda_{1}=V_{B}, \lambda_{2}=-V_{P}$ . So we are able to formulate the main problem of the paper:

To solve the hyperbolic system (4) satisfying initial conditions

$C_{B}(x, 0)=0, C_{P}(x, 0)=C_{P 0}=$ const. $>0, x \in[0, H]$

and the boundary conditions

$$
\begin{aligned}
& C_{B}(0, t)=0, C_{P}(0, t)=C_{P 0}, t \in[0, T] ; \\
& C_{B}(\Lambda, t)=C_{B \Lambda}, C_{P}(\Lambda, t)=C_{P \Lambda}, t \in[0, T] \\
& C_{B}(\Lambda, t)+C_{P}(\Lambda, t)=\kappa(t)<1, \text { where } \\
& \bar{\kappa}=\sup \{\kappa(t): t \in[0, T]\}<1 .
\end{aligned}
$$

\section{SOLUTIONS OF THE EQUATIONS FOR CHARACTERISTICS OF THE HYPERBOLIC SYSTEM}

Prior to formulate an operator corresponding to the mixed problem we consider Cauchy problems for the characteristics (cf. [3]):

$\frac{d x_{B}(\tau)}{d \tau}=V_{B}\left(x_{B}, \tau\right), x_{B}(t)=x$

$\frac{d x_{P}(\tau)}{d \tau}=-V_{P}\left(x_{P}(\tau), \tau\right), x_{P}(\tau)(t)=x$

for each $(x, t) \in \Pi$. Here the characteristic functions $\lambda_{B}(\xi, \tau)=V_{B}(\xi, \tau)>0$ and $\lambda_{P}(\xi, \tau)=-V_{P}(\xi, \tau)<0$ are continuous ones.

We need the following auxiliary assertions:

Lemma 2.1. For every $(x, t) \in \operatorname{int} \Pi$ the initial value problems

$\frac{d x_{B}(\tau)}{d \tau}=V_{B}\left(x_{B}, \tau\right), x_{B}(t)=x$

$\frac{d x_{P}(\tau)}{d \tau}=-V_{P}\left(x_{P}, \tau\right), x_{P}(\tau)(t)=x$

has a unique solution belonging to $\Pi$ provided the velocities to satisfy the inequalities

$\left|V_{B}(u, \tau)-V_{B}(\bar{u}, \tau)\right| \leq L_{B}|u-\bar{u}|$,

$\left|V_{P}(u, \tau)-V_{P}(\bar{u}, \tau)\right| \leq L_{P}|u-\bar{u}|$,

$\left|V_{B}(0, s)\right| \leq \bar{V}_{B} e^{\mu s},\left|V_{P}(0, s)\right| \leq \bar{V}_{P} e^{\mu s}$,

where $L_{B}, L_{P}, \bar{V}_{B}, \bar{V}_{P}, \mu$ are positive constants.

Proof: Let us consider the sets of continuous functions

$M_{B}=\left\{x_{B}(.) \in C[0, T]:\left|x_{B}(\tau)-x\right| \leq X_{B} e^{\mu \tau} \mid\right\}$

$M_{P}=\left\{x_{P}(.) \in C[0, T]:\left|x_{P}(\tau)-x\right| \leq X_{P} e^{\mu \tau} \mid\right\}$

with metrics

$d_{P}(x, \bar{x})=\max \left\{e^{-\mu \tau}\left|x_{P}(\tau)-\bar{x}_{P}(\tau)\right|: \tau \in[0, T]\right\}$, where $T$, $X_{B}, X_{P}$ are prescribed positive constants.

Define the operator $Q=\left(Q_{1}, Q_{2}\right): M_{B} \times M_{P} \rightarrow M_{B} \times M_{P}$ by the formulas:

$Q_{1}\left(x_{B}\right)(\tau):=x+\int_{t}^{\tau} V_{B}\left(x_{B}(s), s\right) d s, t \in[0, T]$ 
$Q_{2}\left(x_{P}\right)(\tau):=x+\int_{t}^{\tau} V_{P}\left(x_{P}(s), s\right) d s, t \in[0, T]$

First we show that the operator $Q_{1}$ maps the set $M_{B}$ into itself. Indeed,

$\left|Q_{1}\left(x_{B}\right)(\tau)-x\right| \leq \int_{t}^{\tau}\left|V_{B}\left(x_{B}(s), s\right)-V_{B}(0, s)\right| d s+\int_{t}^{\tau}\left|V_{B}(0, s)\right| d s \leq$

$\leq L_{B} \int_{t}^{\tau}\left|x_{B}(s)\right| d s+\bar{V}_{B} \int_{t}^{\tau} e^{\mu s} d s \leq L_{B} \int_{t}^{\tau}\left|x_{B}(s)-x\right| d s+L_{B} \int_{t}^{\tau}|x| d s+$

$+\bar{V}_{B} \frac{e^{\mu \tau}}{\mu} \leq L_{B} X_{B} \int_{t}^{\tau} e^{\mu s} d s+L_{B}|x| \int_{t}^{\tau} e^{\mu s} d s+\bar{V}_{B} \frac{e^{\mu \tau}}{\mu} \leq$

$\leq \frac{L_{B} X_{B}+L_{B}|x|+\bar{V}_{B}}{\mu} e^{\mu \tau} \leq X_{B} e^{\mu \tau}$.

The last inequality is satisfied for sufficiently large $\mu>0$.

It remains to show that $Q_{1}\left(x_{B}\right)$ is a contractive operator. Indeed,

$\left|Q_{1}\left(x_{B}\right)(\tau)-Q_{1}\left(\bar{x}_{B}\right)(\tau)\right| \leq\left|\int_{t}^{\tau} V_{B}\left(x_{B}(s), s\right) d s-\int_{t}^{\tau} V_{B}\left(\bar{x}_{B}(s), s\right) d s\right| \leq$

$\leq L_{B} \int_{t}^{\tau} e^{\mu s}\left|x_{B}(s)-\bar{x}_{B}(s)\right| e^{\mu s} d s \leq L_{B} d_{B}\left(x_{B}, \bar{x}_{B}\right) \int_{t}^{\tau} e^{\mu s} d s \leq$

$\leq L_{B} d_{B}\left(x_{B}, \bar{x}_{B}\right) \frac{e^{\mu \tau}}{\mu}$

that implies $d_{B}\left(Q_{1}\left(x_{B}\right), Q_{1}\left(\bar{x}_{B}\right)\right) \leq \frac{L_{B}}{\mu} d_{B}\left(x_{B}, \bar{x}_{B}\right)$.

In a similar way we obtain

$\left|Q_{2}\left(x_{P}\right)(\tau)-x\right| \leq \int_{t}^{\tau}\left|V_{P}\left(x_{P}(s), s\right)-V_{P}(0, s)\right| d s+\int_{t}^{\tau}\left|V_{P}(0, s)\right| d s \leq$

$\leq L_{P} \int_{t}^{\tau}\left|x_{P}(s)\right| d s+\bar{V}_{P} \int_{t}^{\tau} e^{\mu s} d s \leq L_{P} \int_{t}^{\tau}\left|x_{P}(s)-x\right| d s+L_{P} \int_{t}^{\tau}|x| d s+$

$+\bar{V}_{P} \frac{e^{\mu \tau}}{\mu} \leq L_{P} X_{P} \int_{t}^{\tau} e^{\mu s} d s+L_{P}|x| \int_{t}^{\tau} e^{\mu s} d s+\bar{V}_{P} \frac{e^{\mu \tau}}{\mu} \leq$

$\leq \frac{L_{P} X_{P}+L_{P}|x|+\bar{V}_{P}}{\mu} e^{\mu \tau} \leq X_{P} e^{\mu \tau}$

and $d_{P}\left(Q_{2}\left(x_{P}\right), Q_{2}\left(\bar{x}_{P}\right)\right) \leq \frac{L_{P}}{\mu} d_{P}\left(x_{P}, \bar{x}_{P}\right)$.

\section{Consequently}

$d_{B}\left(Q_{1}\left(x_{B}\right), Q_{1}\left(\bar{x}_{B}\right)\right)+d_{P}\left(Q_{2}\left(x_{P}\right), Q_{2}\left(\bar{x}_{P}\right)\right) \leq$

$\leq \frac{L_{P}}{\mu} d_{P}\left(x_{P}, \bar{x}_{P}\right)+\frac{L_{B}}{\mu} d_{B}\left(x_{B}, \bar{x}_{B}\right) \leq$

$\leq \frac{L_{P}+L_{B}}{\mu}\left(d_{P}\left(x_{P}, \bar{x}_{P}\right)+d_{B}\left(x_{B}, \bar{x}_{B}\right)\right)$, that is, $Q$ is contractive operator for sufficiently large

$\mu>0$. The fixed point of $Q$ is a unique solution $\left(x_{B}, x_{P}\right)$ of the above Cauchy problems (6) and (7).

Lemma 3.1 is thus proved.

Denote by $T_{B}(t)=\frac{H}{V_{B}\left(x_{B}(t), t\right)}, T_{P}(t)=\frac{H}{V_{P}\left(x_{P}(t), t\right)}$.

III. AN OPERATOR FORMULATION OF THE MIXED PROBLEM

Let $\left(\varphi_{B}(\tau ; x, t), \varphi_{P}(\tau ; x, t)\right)$ be solutions of (6) and (7).

Denote by $\chi_{B}(x, t)$ the smallest value of $\tau$ such that the solution $\varphi_{B}(\tau ; x, t)$ of (6) still belongs to $\Pi$ and by $\chi_{P}(x, t)$ - the respective value of $\tau$ for the solution $\varphi_{P}(\tau ; x, t)$ of $(7)$.

If $\chi_{B}(x, t)>0$ then $\quad \varphi_{B}\left(\chi_{B}(x, t) ; x, t\right)=0 \quad$ or $\varphi_{B}\left(\chi_{B}(x, t) ; x, t\right)=H$ and respectively if $\chi_{P}(x, t)>0$ then $\varphi_{P}\left(\chi_{P}(x, t) ; x, t\right)=0$ or $\varphi_{P}\left(\chi_{P}(x, t) ; x, t\right)=H$. We notice that $0 \leq \chi_{B}(x, t) \leq t, 0 \leq \chi_{P}(x, t) \leq t$.

Introduce the sets

$D_{i n, B}=\left\{(x, t) \in \Pi: \chi_{B}(x, t)=0\right\}$,

$D_{i n, P}=\left\{(x, t) \in \Pi: \chi_{P}(x, t)=0\right\}$,

$D_{0 B}=\left\{(x, t) \in \Pi: \chi_{B}(x, t)>0, \varphi_{B}\left(\chi_{B}(x, t) ; x, t\right)=0\right\}$,

$D_{H B}=\left\{(x, t) \in \Pi: \chi_{B}(x, t)>0, \varphi_{B}\left(\chi_{B}(x, t) ; x, t\right)=H\right\}=\varnothing$,

$D_{0 P}=\left\{(x, t) \in \Pi: \chi_{P}(x, t)>0, \varphi_{P}\left(\chi_{P}(x, t) ; x, t\right)=0\right\}=\varnothing$,

$D_{H P}=\left\{(x, t) \in \Pi: \chi_{P}(x, t)>0, \varphi_{P}\left(\chi_{P}(x, t) ; x, t\right)=H\right\}$.

We introduce the denotations

$$
\begin{aligned}
\Phi_{B}\left(C_{B}, C_{P}\right)(x, t) & =\left\{\begin{array}{c}
C_{B 0}\left(\chi_{B}(x, t)\right), \quad(x, t) \in D_{i n, B} \\
\Phi_{0 B}\left(C_{B}, C_{P}\right)\left(\chi_{B}(x, t)\right), \quad(x, t) \in D_{0 B}
\end{array}=\right. \\
& =\left\{\begin{array}{cc}
0, & (x, t) \in D_{i n, B} \\
C_{B 0}, & (x, t) \in D_{0 B}
\end{array}\right.
\end{aligned}
$$

and $\Phi_{P}\left(C_{B}, C_{P}\right)(x, t)=\left\{\begin{array}{c}C_{P 0}\left(\chi_{P}(x, t)\right), \quad(x, t) \in D_{i n, P} \\ \kappa(t)-C_{P}\left(H, \chi_{P}(x, t)\right), \quad(x, t) \in D_{0 P}\end{array}\right.$

So we assign to the above mixed problem the following system of operator equations 


$$
\begin{aligned}
& \frac{\partial C_{B}(x, t)}{\partial t}=k_{1} C_{P}(x, t)-k_{2} C_{B}(x, t)-V_{B}(x, t) \frac{\partial C_{B}(x, t)}{\partial x}- \\
& -C_{B} \frac{\partial V_{B}(x, t)}{\partial x} \\
& \frac{\partial C_{P}(x, t)}{\partial t}=-k_{1} C_{P}(x, t)+k_{2} C_{B}(x, t)+ \\
& +V_{P}(x, t) \frac{\partial C_{P}(x, t)}{\partial x}+C_{P} \frac{\partial V_{P}(x, t)}{\partial x} \\
& C_{B}(x, t)=0,(x, t) \in D_{i n, B} \\
& C_{B}(x, t)=C_{B 0}+\int_{\chi_{B}(x, t)}^{t}\left(k_{1} C_{B}\left(\chi_{B}(x, s), s\right)-\right. \\
& \left.-k_{2} C_{B}\left(\chi_{B}(x, s), s\right)-C_{B} \frac{\partial V_{B}(x, s)}{\partial x}\right) d s,(x, t) \in D_{0 B} \\
& C_{P}(x, t)=C_{P 0},(x, t) \in D_{i n, P} \\
& C_{P}(x, t)=\kappa(t)-C_{B}\left(H, \chi_{P}(x, t)\right)+\int_{\chi_{P}(x, t)}^{t}\left(-k_{1} C_{B}\left(\chi_{P}(x, s), s\right)+\right. \\
& \left.+k_{2} C_{P}\left(\chi_{P}(x, s), s\right)+C_{P} \frac{\partial V_{P}(x, s)}{\partial x}\right) d s,(x, t) \in D_{0 P} .
\end{aligned}
$$

Introduce the function sets:

$M_{B}=\left\{C_{B} \in C[0, H] \times\left[0, T_{0}\right]:\left|C_{B}(x, t)\right| \leq \hat{C}_{B} e^{\mu t}, x \in[0, H]\right\}$, $M_{P}=\left\{C_{P} \in C[0, H] \times\left[0, T_{0}\right]:\left|C_{P}(x, t)\right| \leq \hat{C}_{P} e^{\mu t}, x \in[0, H]\right\}$,

where $\hat{C}_{B}, \hat{C}_{P}$ and $\mu$ are positive constants.

It is easy to verify that the set $M_{B} \times M_{P}$ turns out into a complete metric space with respect to the metric:

$$
\rho\left(\left(C_{B}, C_{P}\right),\left(\bar{C}_{B}, \bar{C}_{P}\right)\right)=\max \left\{\rho\left(C_{B}, \bar{C}_{B}\right), \rho\left(C_{P}, \bar{C}_{P}\right)\right\},
$$

where

$$
\begin{aligned}
& \rho\left(C_{B}, \bar{C}_{B}\right)= \\
& \quad=\sup \left\{e^{-\mu t}\left|C_{B}(x, t)-\bar{C}_{B}(x, t)\right|:(x, t) \in[0, H] \times[0, T]\right\}, \\
& \rho\left(C_{P}, \bar{C}_{P}\right)= \\
& \quad=\sup \left\{e^{-\mu t}\left|C_{P}(x, t)-\bar{C}_{P}(x, t)\right|:(x, t) \in[0, H] \times[0, T]\right\} .
\end{aligned}
$$

Now we define an operator

$T=\left(T_{B}, T_{P}\right): M_{B} \times M_{P} \rightarrow M_{B} \times M_{P}$ by the formulas

$T_{B}\left(C_{B}, C_{P}\right)(x, t):=0,(x, t) \in D_{i n, B}$,

$$
\begin{aligned}
& T_{B}\left(C_{B}, C_{P}\right)(x, t):=C_{B 0}+\int_{\chi_{B}(x, t)}^{t}\left(k_{1} C_{B}\left(\chi_{B}(x, s), s\right)-\right. \\
& \left.-k_{2} C_{P}\left(\chi_{B}(x, s), s\right)-C_{B} \frac{\partial V_{B}\left(\chi_{B}, s\right)}{\partial x}\right) d s,(x, t) \in D_{0 B} ; \\
& T_{P}\left(C_{B}, C_{P}\right)(x, t):=C_{0 P},(x, t) \in D_{i n, P}, \\
& T_{P}\left(C_{B}, C_{P}\right)(x, t):=\kappa(t)-C_{B}\left(H, \chi_{P}(x, t)\right)+ \\
& +\int_{\chi_{P}(x, t)}^{t}\left(-k_{1} C_{B}\left(\chi_{B}(x, s), s\right)+k_{2} C_{P}\left(\chi_{P}(x, s), s\right)+\right. \\
& \left.+C_{P} \frac{\partial V_{P}\left(\chi_{P}, s\right)}{\partial x}\right) d s,(x, t) \in D_{0 P} .
\end{aligned}
$$

\section{EXISTENCE-UNIQUENESS THEOREM}

We call a generalized solution $\left(C_{B}, C_{P}\right)$ of (4), (5) if $\left(C_{B}, C_{P}\right)$ is a solution of operator equations (8). The main purpose of the section is to prove an existence-uniqueness of solution of (8).

Theorem 1. Let the following conditions be fulfilled for sufficiently large $\mu>0$ :

1) $\left|\frac{\partial V_{B}(x, s)}{\partial x}\right| \leq \bar{V}_{B x} e^{\mu s} ; \quad\left|\frac{\partial V_{P}(x, s)}{\partial x}\right| \leq \bar{V}_{P x} e^{\mu s}$;

2) $\frac{\left(1+k_{1}\right) \hat{C}_{B}+k_{2} \hat{C}_{P}+\hat{C}_{B} \bar{V}_{B x}}{\mu} \leq \hat{C}_{B}$;

3) $\hat{\kappa}+\hat{C}_{P} e^{-\mu \chi_{0}}+\frac{k_{1} \hat{C}_{B}+k_{2} \hat{C}_{P}+\hat{C}_{P} \bar{V}_{P x}}{\mu} \leq \hat{C}_{P}$;

4) $e^{-\mu \chi_{0}}+\frac{k_{1}+k_{2}}{\mu}<1 ;$ 5) $C_{B 0} \leq \hat{C}_{B} ; C_{0 P} \leq \hat{C}_{P}$;

6) $\hat{\kappa}=\sup \{\kappa(t): t \in[0, T]\}<1$.

Then there exists a unique generalized solution of (8) on the set $[0, H-\varepsilon] \times[0, T]$, where $0<\varepsilon<H$.

Remark 4.1. It is easy to see that $t-\chi_{P} \geq \chi_{0}$, $[0, H-\varepsilon] \times[0, T]$.

Proof: We show that the operator $T=\left(T_{B}, T_{P}\right): M_{B} \times M_{P} \rightarrow M_{B} \times M_{P}$ above introduced maps the set $M_{B} \times M_{P}$ into itself.

We notice that $T_{B}\left(C_{B}, C_{P}\right)(x, t)$ and $T_{P}\left(C_{B}, C_{P}\right)(x, t)$ are continuous functions. First we have to show that

$\left|T_{B}\left(C_{B}, C_{P}\right)(x, t)\right| \leq \hat{C}_{B} e^{\mu t},\left|T_{P}\left(C_{B}, C_{P}\right)(x, t)\right| \leq \hat{C}_{P} e^{\mu t}$.

Indeed, $\left|\Phi_{B}\left(C_{B}, C_{P}\right)(x, t)\right|=0$ and therefore 


$$
\begin{gathered}
\left|T_{B}\left(C_{B}, C_{P}\right)(x, t)\right| \leq C_{B 0}+\int_{\chi_{B}}^{t}\left(k_{1}\left|C_{B}\left(\chi_{B}, s\right)\right|+k_{2}\left|C_{P}\left(\chi_{B}, s\right)\right|+\right. \\
\left.+\left|C_{B}\left(\chi_{B}, s\right)\right|\left|\frac{\partial V_{B}\left(\chi_{B}, s\right)}{\partial x}\right|\right) d s \leq\left(\left(1+k_{1}\right) \hat{C}_{B}+k_{2} \hat{C}_{P}+\hat{C}_{B} \bar{V}_{B x}\right) . \\
\cdot \int_{\chi_{B}}^{t} e^{\mu s} d s \leq\left(\left(1+k_{1}\right) \hat{C}_{B}+k_{2} \hat{C}_{P}+\hat{C}_{B} \bar{V}_{B x}\right) \frac{e^{\mu t}-e^{\mu \chi_{B}}}{\mu} \leq \\
\leq \frac{\left(1+k_{1}\right) \hat{C}_{B}+k_{2} \hat{C}_{P}+\hat{C}_{B} \bar{V}_{B x}}{\mu} e^{\mu t} \leq \hat{C}_{B} e^{\mu t} ; \\
\left|T_{P}\left(C_{B}, C_{P}\right)(x, t)\right| \leq \kappa(t)+C_{B}\left(H, \chi_{P}(x, t)\right)+\int_{\chi_{P}}^{t}\left(k_{1}\left|C_{B}\left(\chi_{P}, s\right)\right|+\right. \\
\left.+k_{2}\left|C_{P}\left(\chi_{p}, s\right)\right|+\left|C_{P}\left(\chi_{P}, s\right)\right| \frac{\partial V_{P}\left(\chi_{P}, s\right)}{\partial x} \mid\right) d s \leq \hat{\kappa}^{\mu t}+\hat{C}_{P} e^{\mu \chi_{P}}+ \\
+\frac{k_{1} \hat{C}_{B}+k_{2} \hat{C}_{P}+\hat{C}_{P} \bar{V}_{P x} e^{\mu t} \leq\left(\hat{\kappa}+\hat{C}_{P} e^{-\mu\left(t-\chi_{P}\right)}+\right.}{\mu} \\
\left.+\frac{k_{1} \hat{C}_{B}+k_{2} \hat{C}_{P}+\hat{C}_{P} \bar{V}_{P x}}{\mu}\right) e^{\mu t} \leq \\
\leq\left(\hat{\kappa}+\hat{C}_{P} e^{-\mu \chi_{0}}+\frac{k_{1} \hat{C}_{B}+k_{2} \hat{C}_{P}+\hat{C}_{P} \bar{V}_{P x}}{\mu}\right) e^{\mu t} \leq \hat{C}_{P} e^{\mu t}
\end{gathered}
$$

for sufficiently large $\mu>0$.

The operator $T$ is a contractive one. Indeed,

$$
\begin{aligned}
& \left|T_{B}\left(C_{B}, C_{P}\right)(x, t)-T_{B}\left(\bar{C}_{B}, \bar{C}_{P}\right)(x, t)\right| \leq \\
& \leq \int_{\chi_{B}}^{t}\left(k_{1}\left|C_{B}\left(\chi_{B}, s\right)-\bar{C}_{B}\left(\chi_{B}, s\right)\right|+k_{2}\left|C_{P}\left(\chi_{B}, s\right)-\bar{C}_{P}\left(\chi_{B}, s\right)\right|+\right. \\
& \left.+\left|C_{B}\left(\chi_{B}, s\right)-\bar{C}_{B}\left(\chi_{B}, s\right)\right|\left|\frac{\partial V_{B}\left(\chi_{B}, s\right)}{\partial x}\right|\right) d s \leq \rho\left(C_{B}, \bar{C}_{B}\right) k_{1} . \\
& \cdot \int_{\chi_{B}}^{t} e^{\mu s} d s+\rho\left(C_{P}, \bar{C}_{P}\right) k_{2} \int_{\chi_{B}}^{t} e^{\mu s} d s+\rho\left(C_{B}, \bar{C}_{B}\right) \bar{V}_{B x} \int_{\chi_{B}}^{t} e^{\mu s} d s \leq \\
& \leq \max \left\{\rho\left(C_{B}, \bar{C}_{B}\right), \rho\left(C_{P}, \bar{C}_{P}\right)\right\}\left(k_{1}+k_{2}+\bar{V}_{B x}\right) \frac{e^{\mu t}-e^{\mu\left(t-\chi_{B}\right)}}{\mu} \leq \\
& \leq e^{\mu t} \rho\left(\left(C_{B}, C_{P}\right),\left(\bar{C}_{B}, \bar{C}_{P}\right)\right) \frac{k_{1}+k_{2}+\bar{V}_{B x}}{\mu} .
\end{aligned}
$$

\section{Consequently}

$$
\begin{aligned}
& \rho\left(T_{B}\left(C_{B}, C_{P}\right), T_{B}\left(\bar{C}_{B}, \bar{C}_{P}\right)\right) \leq \\
& \quad \leq \frac{k_{1}+k_{2}+\bar{V}_{B x}}{\mu} \rho\left(\left(C_{B}, C_{P}\right),\left(\bar{C}_{B}, \bar{C}_{P}\right)\right) .
\end{aligned}
$$

For the second component, we have
$\left|T_{P}\left(C_{B}, C_{P}\right)(x, t)-T_{P}\left(\bar{C}_{B}, \bar{C}_{P}\right)(x, t)\right| \leq$ $\leq\left|C_{B}\left(H, \chi_{P}(x, t)\right)-\bar{C}_{B}\left(H, \chi_{P}(x, t)\right)\right| \leq$ $\leq \int_{\chi_{P}}^{t}\left(k_{1}\left|C_{B}\left(\chi_{P}, s\right)-\bar{C}_{B}\left(\chi_{P}, s\right)\right|+k_{2}\left|C_{P}\left(\chi_{P}, s\right)-\bar{C}_{P}\left(\chi_{P}, s\right)\right|+\right.$ $\left.+\left|C_{P}\left(\chi_{P}, s\right)-\bar{C}_{P}\left(\chi_{P}, s\right)\right|\left|\frac{\partial V_{P}\left(\chi_{P}, s\right)}{\partial x}\right|\right) d s \leq \rho\left(C_{B}, \bar{C}_{B}\right) e^{\mu \chi_{P}}+$ $+\rho\left(C_{B}, \bar{C}_{B}\right) k_{1} \int_{\chi_{P}}^{t} e^{\mu s} d s+\rho\left(C_{P}, \bar{C}_{P}\right) k_{2} \int_{\chi_{P}}^{t} e^{\mu s} d s+\rho\left(C_{P}, \bar{C}_{P}\right)$. $\bar{V}_{P_{x}} \int_{\chi_{P}}^{t} e^{\mu s} d s \leq e^{\mu t} \rho\left(C_{B}, \bar{C}_{B}\right) e^{-\mu\left(t-\chi_{P}\right)}+\rho\left(C_{B}, \bar{C}_{B}\right) k_{1} \int_{\chi_{P}}^{t} e^{\mu s} d s+$ $+\rho\left(C_{P}, \bar{C}_{P}\right) k_{2} \int_{\chi_{P}}^{t} e^{\mu s} d s+\rho\left(C_{P}, \bar{C}_{P}\right) \bar{V}_{P_{x}} \int_{\chi_{P}}^{t} e^{\mu s} d s \leq$ $\leq e^{\mu t} \rho\left(C_{B}, \bar{C}_{B}\right) e^{-\mu \chi_{0}}+\frac{e^{\mu t}-e^{\mu \chi_{P}}}{\mu}$.

$$
\begin{aligned}
& .\left(\rho\left(C_{B}, \bar{C}_{B}\right) k_{1}+\rho\left(C_{P}, \bar{C}_{P}\right) k_{2}+\rho\left(C_{P}, \bar{C}_{P}\right) \bar{V}_{P x}\right) \leq \\
& \quad \leq e^{\mu t}\left(e^{-\mu \chi_{0}}+\frac{k_{1}}{\mu}+\frac{k_{2}}{\mu}+\frac{\bar{V}_{P x}}{\mu}\right) \rho\left(\left(C_{B}, C_{P}\right),\left(\bar{C}_{B}, \bar{C}_{P}\right)\right)
\end{aligned}
$$

which implies

$$
\begin{aligned}
\rho\left(T_{P}\left(C_{B}, C_{P}\right)\right. & \left., T_{P}\left(\bar{C}_{B}, \bar{C}_{P}\right)\right) \leq \\
& \leq\left(e^{-\mu \frac{\varepsilon}{V_{P}}}+\frac{k_{1}+k_{2}}{\mu}\right) \rho\left(\left(C_{B}, C_{P}\right),\left(\bar{C}_{B}, \bar{C}_{P}\right)\right) .
\end{aligned}
$$

\section{Since}

$$
\begin{aligned}
& \rho\left(T_{B}\left(C_{B}, C_{P}\right), T_{B}\left(\bar{C}_{B}, \bar{C}_{P}\right)\right) \leq \\
& \leq\left(e^{-\mu \chi_{0}}+\frac{k_{1}+k_{2}+\bar{V}_{P_{X}}}{\mu}\right) \rho\left(\left(C_{B}, C_{P}\right),\left(\bar{C}_{B}, \bar{C}_{P}\right)\right)
\end{aligned}
$$

and

$$
\begin{aligned}
& \rho\left(T_{P}\left(C_{B}, C_{P}\right), T_{P}\left(\bar{C}_{B}, \bar{C}_{P}\right)\right) \leq \\
& \quad \leq \rho\left(\left(C_{B}, C_{P}\right),\left(\bar{C}_{B}, \bar{C}_{P}\right)\right)\left(e^{-\mu \frac{\varepsilon}{V_{P}}}+\frac{k_{1}+k_{2}}{\mu}\right),
\end{aligned}
$$

it follows

$$
\begin{gathered}
\max \left\{\rho\left(T_{B}\left(C_{B}, C_{P}\right), T_{B}\left(\bar{C}_{B}, \bar{C}_{P}\right)\right), \rho\left(T_{P}\left(C_{B}, C_{P}\right), T_{P}\left(\bar{C}_{B}, \bar{C}_{P}\right)\right)\right\} \leq \\
\leq\left(e^{-\mu \chi_{0}}+\frac{k_{1}+k_{2}+\bar{V}_{P x}}{\mu}\right) \rho\left(\left(C_{B}, C_{P}\right),\left(\bar{C}_{B}, \bar{C}_{P}\right)\right)
\end{gathered}
$$

that is,

$$
\begin{aligned}
\rho\left(\left(T_{B}\left(C_{B}, C_{P}\right), T_{P}\left(C_{B}, C_{P}\right)\right),\left(T_{P}\left(\bar{C}_{B}, \bar{C}_{P}\right), T_{P}\left(\bar{C}_{B}, \bar{C}_{P}\right)\right)\right) \leq \\
\leq\left(e^{-\mu \chi_{0}}+\frac{k_{1}+k_{2}+\bar{V}_{P X}}{\mu}\right) \rho\left(\left(C_{B}, C_{P}\right),\left(\bar{C}_{B}, \bar{C}_{P}\right)\right)
\end{aligned}
$$


and in this way, we have shown that $T$ is a contractive operator. The fixed point of $T$ is a solution of the mixed problem above formulated.

The main Theorem is thus proved.

\section{CONCLUSION REMARKS}

Here we show the process of obtaining of successive approximations. We begin with the first step choosing $C_{B}^{(0)}(x, t)=C_{B 0}, C_{P}^{(0)}(x, t)=C_{P 0}$. Then

$$
\begin{aligned}
& C_{B}^{(n+1)}(x, t)=C_{B 0}+\int_{\chi_{B}}^{t}\left(k_{1} C_{B}^{(n)}\left(\chi_{B}(x, s), s\right)-\right. \\
& \left.\quad-k_{2} C_{P}^{(n)}\left(\chi_{B}(x, s), s\right)-C_{B}^{(n)} \frac{\partial V_{B}\left(\chi_{B}, s\right)}{\partial x}\right) d s,(x, t) \in D_{0 B} \\
& C_{P}^{(n+1)}(\Lambda, t)=\kappa(t)-C_{B}^{(n)}\left(\Lambda, \chi_{P}(x, t)\right)+\int_{\chi_{B}}^{t}\left(-k_{1} C_{B}^{(n)}\left(\chi_{P}(x, s), s\right)+\right. \\
& \left.+k_{2} C_{P}^{(n)}\left(\chi_{P}(x, s), s\right)+C_{P}^{(n)} \frac{\partial V_{P}\left(\chi_{P}, s\right)}{\partial x}\right) d s,(x, t) \in D_{0 P} ; \\
& n=0,1,2, \ldots
\end{aligned}
$$

\section{REFERENCES}

[1] D. D. Parashkevova. (November 2020). On the qualitative analysis of certain flotation processes. IJESRT. 9(11). pp. 91-99. DOI: https://doi.org/10.29121/ijesrt.v9.i11.2020.12.

[2] Yu. B. Rubinstein, Yu. A. Filippov, Flotation Kinetics, Moscow, 1980 (in Russian).

[3] V. G. Angelov, A Method for Analysis of Transmission Lines Terminated by Nonlinear Loads. Nova Science, New York, 2014.

[4] V. G. Angelov, Lossy transmission lines with Josephson junction continuous generalized solutions. Communication in Applied Analysis, 20 (2016), pp. 91-106.

[5] V. G. Angelov, Polynomial Nonlinearity in Superconducting Lossless Transmission Line Equations. Journal of Robotic and Mechatronic Systems, vol.1, No.1, (2016), pp. 1-9.

[6] V. G. Angelov. (October 2019). Nonlinear Membrane Circuit Loaded on a Lossy Transmission Line without the Heaviside's Condition. European Journal of Engineering Research and Science. 4(10). pp. 190-197. DOI: https://doi.org/10.24018/ejers.2019.4.10.1583.

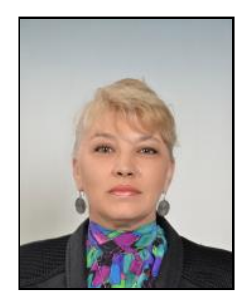

Daniela D. Parashkevova is a Assoc. Prof. PhD, Department of Automation of Mining Production, Faculty of Mining Electromechanics, University of Mining and Geology „St. I. Rilski” in Sofia, Bulgaria.

She is a lecturer in the Department of Automation of Mining Production. In the $\mathrm{PhD}$ Thesis entitled „Analysis of Technological Solutions and Methods for Mineral Processing Control of Copper Ores" a systematic approach for distributed control of multidimensional flotation processes with hybrid nature is developed. It is based on the theory of large systems using multivalued logical probability fuzzy functions. Analysis, algorithms, methods, and control system structures for flotation processes are proposed, possessing numerical intelligence. The methods proposed by mathematical data processing of big dates are justified using results from the flotation process in the flotation factory Ellatzite-Med $\mathrm{AD}$, Bulgaria. They are in 30 applications outlined. 